Published in final edited form as:

J Clin Virol. 2014 August ; 60(4): 392-398. doi:10.1016/j.jcv.2014.05.005.

\title{
Measures of Viral Load using Abbott Real-Time HIV-1 Assay on Venous and Fingerstick Dried Blood Spots from Provider- Collected Specimens in Malawian District Hospitals
}

\author{
Sarah E. Rutstein ${ }^{1,2}$, Deborah Kamwendo ${ }^{3}$, Lebah Lugali ${ }^{4}$, Isaac Thengolose ${ }^{3}$, Gerald \\ Tegha $^{3}$, Susan A. Fiscus ${ }^{5}$, Julie A. E. Nelson ${ }^{5}$, Mina C. Hosseinipour ${ }^{2,3}$, Abdoulaye Sarr $^{6}$, \\ Sundeep Gupta ${ }^{6}$, Frank Chimbwandira ${ }^{7}$, Reuben Mwenda ${ }^{7}$, and Ronald Mataya ${ }^{8}$ \\ ${ }^{1}$ Department of Health Policy and Management, University of North Carolina at Chapel Hill, \\ Chapel Hill, North Carolina \\ ${ }^{2}$ Department of Medicine, University of North Carolina at Chapel Hill, Chapel Hill, North Carolina \\ ${ }^{3}$ UNC Project, Lilongwe, Malawi \\ ${ }^{4}$ Johns Hopkins University, University of North Carolina at Chapel Hill, Chapel Hill, North Carolina \\ ${ }^{5}$ UNC Center for AIDS Research and Department of Microbiology and Immunology, University of \\ North Carolina at Chapel Hill, Chapel Hill, North Carolina \\ ${ }^{6}$ Centers for Disease Control, Malawi \\ ${ }^{7}$ Ministry of Health, Lilongwe, Malawi \\ ${ }^{8}$ School of Public Health, Loma Linda University
}

\section{Abstract}

Background-Viral suppression is a key indicator of antiretroviral therapy (ART) response among HIV-infected patients. Dried blood spots (DBS) are an appealing alternative to conventional plasma-based virologic testing, improving access to monitoring in resource-limited settings. However, validity of DBS obtained from fingerstick in field settings remains unknown.

Objectives-Investigate feasibility and accuracy of DBS vs plasma collected by healthcare workers in real-world settings of remote hospitals in Malawi. Compare venous DBS to fingerstick DBS for identifying treatment failure.

Study design-We recruited patients from ART clinics at two district hospitals in Malawi, collecting plasma, venous DBS (vDBS), and fingerstick DBS (fsDBS) cards for the first 149 patients, and vDBS and fsDBS only for the subsequent 398 patients. Specimens were tested using

(C) 2014 Elsevier B.V. All rights reserved.

correspondence:srutstein@unc.edu.

Publisher's Disclaimer: This is a PDF file of an unedited manuscript that has been accepted for publication. As a service to our customers we are providing this early version of the manuscript. The manuscript will undergo copyediting, typesetting, and review of the resulting proof before it is published in its final citable form. Please note that during the production process errors may be discovered which could affect the content, and all legal disclaimers that apply to the journal pertain. 
Abbott RealTime HIV-1 Assay (lower detection limit 40 copies/ml (plasma) and 550 copies $/ \mathrm{ml}$ (DBS)).

Results-21/149 (14.1\%) had detectable viremia (>1.6 log copies/ml), 13 of which were detectable for plasma, vDBS, and fsDBS. Linear regression demonstrated high correlation for plasma vs. DBS (vDBS: $\beta=1.19, \mathrm{R}^{2} 0.93(\mathrm{p}<0.0001)$; fsDBS $\beta=1.20, \mathrm{R}^{2} 0.90(\mathrm{p}<0.0001)$ ) and vDBS vs. fsDBS $\left(\beta=0.88, \mathrm{R}^{2} 0.73\right.$, $(\mathrm{p}<0.0001)$ ). Mean difference between plasma and vDBS was $0.51 \mathrm{log}$ copies/ml [SD: 0.33 ] and plasma and fsDBS $0.46 \mathrm{log}$ copies/ml [SD: 0.30]. At 5000 copies/ml, sensitivity was $100 \%$, and specificity was $98.6 \%$ and $97.8 \%$ for vDBS and fsDBS, respectively, compared to plasma.

Conclusions-DBS from venipuncture and fingerstick perform well at the failure threshold of 5000 copies/ml. Fingerstick specimen source may improve access to virologic treatment monitoring in resource-limited settings given task-shifting in high-volume, low-resource facilities.

\section{Background}

HIV viral suppression is a key indicator of successful treatment for HIV-infected patients on antiretroviral therapy (ART). Global treatment access initiatives have resulted in millions receiving life-saving therapy in resource-limited settings. With over 9.7 million people receiving ART in low- and middle-income countries, the issue of how to appropriately monitor patients is now an urgent international issue. ${ }^{1-3}$ Feasible treatment monitoring strategies in these settings remain unclear.

The expense and complexity of conventional plasma-based viral load (VL) tests limit their usefulness in resource-limited settings ${ }^{4}$. The sensitivity of immunologic and clinical staging for identifying treatment failure is highly variable and generally $1 \mathrm{low}^{5-15}$, thus the utility of these approaches in driving treatment decisions has been $\operatorname{mixed}^{5,6,8,9,11,16-25}$. Point-ofcare VL tests are under evaluation, but effective and affordable point-of-care technologies remain unavailable ${ }^{26}$.

Use of dried blood spots (DBS) alleviates technological and cold-chain barriers, enabling access to VL monitoring in remote settings and potentially improving identification of ART failure $^{27,28}$. DBS from fingersticks should decrease associated costs compared to venous DBS, task-shifting to lower-level providers and reducing consumable-associated expenses. Fingerstick DBS may also expand monitoring to health centers without phlebotomy capabilities $^{29}$. Previous CD4 test evaluations have shown mixed results in terms of concordance between venous and capillary specimens ${ }^{30,31}$. Numerous studies have suggested the opportunity for venous DBS for VL monitoring $27,29,32-44$ but ours was one of the first tests of fingerstick DBS under true field conditions. We relied on existing clinic personnel for specimen collection, card preparation, and transport to the central laboratory, reflecting a more "real-world" scenario of VL monitoring using DBS via fingerstick.

\section{Objectives}

We investigated the feasibility and accuracy of using DBS in remote district hospitals in Malawi, compared to plasma. We compared venous DBS to fingerstick DBS. This 
validation is a component of an on-going public health evaluation of feasibility and acceptability of DBS in Malawi.

\section{Study Design}

\section{Study population}

Patients were recruited from ART clinics at two district hospitals in central and southern

Malawi. Eligibility criteria included: $\geq 18$ years and on first-line ART for 6 months, 24 months, or any 24-month period thereafter consistent with Malawi recommendations for VL monitoring. Patients were also eligible if they were on therapy $\succeq 6$ months and showing signs of clinical failure (WHO Stage 3 or 4 condition). The first 149 patients had venipuncture collected for plasma and venous DBS (vDBS) card preparation, and fingerstick for DBS (fsDBS) card preparation. The subsequent 398 patients had vDBS and fsDBS cards prepared. This study was approved by the National Health Sciences Research Committee of Malawi, and the Biomedical Institutional Review Board at University of North Carolina, Chapel Hill.

\section{Plasma preparation}

Plasma was collected by venipuncture into an EDTA collection tube. The specimen was processed on-site within 8 hours of collection. Plasma was double-spun at $800 \times \mathrm{g}$ for 10 minutes and stored at $-20 \mathrm{C}$ until shipping.

\section{Card preparation}

Sites were provided DBS collection kits containing: Munktell TFN DBS card with perforated discs ${ }^{45}, 2-3$ capillary tubes, powder-free gloves, sterile lancet, alcohol swab, plastic zip bag, and desiccant. Each DBS card was prepared using a separate kit. Specimens were collected by ART clinic staff: venipuncture was done by laboratory technicians, nurses or clinical officers, and fingerstick by laboratory technicians, nurses, clinical officers, or health-surveillance assistants (HSA). Sites chose which provider collected the specimens based on optimal clinic flow. Cards were labeled with ART patient identifier, source of sample (venous/fingerstick), date/time of collection, and staff initials. Venous blood was collected into an EDTA tube, transferred into 50 $\mu$ capillary tube to facilitate consistent and reliable blood volume per spot, and the contents spotted onto the DBS card. This process was repeated 5 times, allowing for reserve spots in the event of a failed run. Fingerstick specimens were obtained using the sterile lancet after cleaning the finger with alcohol. Blood from fingerstick puncture sites were collected into a 50 $\mathrm{\mu l}$ capillary tube and spotted onto the DBS card, filling 5 circles with $50 \mu \mathrm{l}$ of blood. Cards were air dried at room temperature for at least 3 hours. Dried cards were transferred to individual zip bags with three desiccant sachets and stored at room temperature in the specimen collection room within the clinic or in the laboratory. Sites were instructed to keep cards out of direct sunlight. Storage temperature was not monitored.

\section{Transport to central laboratory}

DBS and plasma specimens were transported to the central laboratory approximately weekly. The internationally monitored research laboratory is located in the capital city 
(Lilongwe). Plasma samples were transported frozen on ice packs or dry ice. DBS cards were transported at ambient temperature. Transport of plasma specimens was completed in one-day ( $250 \mathrm{~km}$ or 3-4 hours from the central region hospital; 350km or 6 hours from southern region hospital). DBS cards were either driven directly by study staff or shipped via in-country specimen shipment arriving within 2-3 days.

\section{VL quantification}

DBS and plasma specimens were tested using the Abbott RealTime HIV-1 Assay (Abbott Laboratories, Chicago, IL) (m2000) (reportable range of 40 to 10,000,000 copies/ml for plasma and lower limit of detection for open-mode DBS protocol of $550 \mathrm{copies} / \mathrm{ml}$. Lower limit of detection for open-mode protocol was determined by previous studies demonstrating DBS VL detectable among $95.7 \%$ of plasma specimens $>550$ copies $/ \mathrm{ml})^{32}$. Using a sterile pipette tip, two DBS spots were punched directly into a tube containing $1.7 \mathrm{~mL} m \mathrm{Lysis}$ buffer. The tube was placed on the mixer for 30 minutes. DBS samples were extracted using the automated m2000sp instrument and amplified according to the $1 \mathrm{~mL}$ DBS protocol. Plasma specimens were tested using the $0.6 \mathrm{~mL}$ plasma protocol.

\section{Statistical analysis}

All RNA values are reported as copies or $\log _{10}$-transformed copy numbers per $\mathrm{ml}$ of blood. Undetectable results ( $<1.60 \mathrm{log}$ copies/ml (plasma), $<2.74 \log$ copies/ml (DBS)) were replaced with the midpoint value between 0 and lower limit of detection (1.3 log copies $/ \mathrm{ml}$ (plasma), $2.44 \log$ copies/ml (DBS)). Sensitivity, specificity, positive predictive value (PPV), and negative predictive value (NPV) were calculated at 5,000 (3.70 log) copies/mlthe current Malawi virological failure definition - and 1000 (3.0 log) copies $/ \mathrm{ml}$, the current WHO plasma-based virological failure threshold ${ }^{46}$. Linear regression analyses were performed comparing results across specimen types. The correlation was expressed as the regression coefficient and $\mathrm{R}$ value. Given non-normal distributions, mean values of VLs between plasma/vDBS, plasma/fsDBS, and vDBS/fsDBS were compared using nonparametric Wilcoxon matched-pairs signed-ranks. Concordance between plasma/vDBS, plasma/fsDBS, and vDBS/fsDBS was assessed using the Bland and Altman test. All analyses were performed using STATA statistical software (version 13.0; StataCorp, College Station, TX). Differences were considered significant when $p$-values were $<0.05$.

\section{Results}

\section{Study Participants}

547 ART patients (mean age 42, standard deviation [SD]10.0) were enrolled (Table 1). 149 plasma/vDBS/fsDBS ("triplicate specimens") and 398 vDBS/fsDBS specimens were obtained.

\section{Turnaround time}

Mean days from specimen collection to laboratory receipt was 8.3 (SD: 4.9; range: 1-28) for plasma and 10.2 (SD: 5.5; range: 1-28) for DBS. Mean days between specimen collection and testing was 12.2 (SD: 4.7; range: 4-25) for plasma, and 17 (SD: 7.2; range: 1-31) for DBS. 


\section{Comparison of plasma to vDBS and fsDBS}

Agreement between plasma/vDBS and plasma/fsDBS was $96.0 \%$ and $91.9 \%$, respectively (Tables $2 \mathrm{a} \& 2 \mathrm{~b}$ ). Among 149 plasma specimens, 21 (14.1\%) samples had detectable VL: 8 (38.1\%) had VL between 1.30 and $2.74 \mathrm{log}$ copies/ml; 3 (14.3\%) had VL between 2.74 and $3.70 \log$ copies $/ \mathrm{ml}$; and 10 (47.6\%) had VL >3.70 log copies/ml. Among patients with a detectable VL, median VL was 3.52 [interquartile range (IQR): 2.04-4.42], 4.51 [3.78 5.27], and 4.11 [3.29 - 5.17] log copies/ml for plasma, vDBS, and fsDBS, respectively. Thirteen triplicates had detectable VL for all three specimen types (Table 3).

Linear regression analyses demonstrated high correlation between plasma VL and DBS [vDBS: $\beta=1.19, \mathrm{R}^{2} 0.93$ ( $\mathrm{p}<0.0001$ ); fsDBS $\beta=1.20, \mathrm{R}^{2} 0.90$ ( $\mathrm{p}<0.0001$ ) (Figure 1a and 1b)]. Mean difference between plasma and vDBS was $1.1 \mathrm{log}$ copies/ml [SD: 0.27] and plasma and fsDBS $1.1 \log$ copies/ml [SD: 0.31] (Figure 2a and 2b). Differences were statistically significant by the Wilcoxon signed rank test $(\mathrm{p}<0.001)$.

Sensitivity of vDBS and fsDBS was 100\% at the 1000 copies/ml threshold (3.0 log copies/ $\mathrm{ml}$ ), and specificity was $97.1 \%$ and $94.9 \%$, respectively, compared to plasma. Prevalence of failure at 1000 copies $/ \mathrm{ml}$ was $8.1 \%$, corresponding to a PPV of $75.0 \%$ and $63.2 \%$ for vDBS and fsDBS, respectively, and 100\% NPV. At a cutoff of 5000 copies $/ \mathrm{ml}$ (3.7 log copies $/ \mathrm{ml}$ ), sensitivity remained $100 \%$ and specificity increased to $98.6 \%$ and $97.8 \%$ for vDBS and fsDBS, respectively, compared to plasma. Despite a lower failure prevalence (6.7\%) PPV improved $(83.3 \%$ and $76.9 \%$ for vDBS and fsDBS), while NPV remained $100 \%$.

At a 5000 copies/ml threshold, vDBS and fsDBS would yield the correct clinical decision $98.7 \%$ and $98.0 \%$ of the time, respectively. We observed 3 clinically discordant triplicates in which DBS results indicated VL $>5000$ copies/ml (i.e., failure) but plasma results were $<5000$ copies/ml (Table 4). Using a lower threshold for defining failure (1000 copies/ml), the rate of clinical discordancy of DBS compared to plasma increased (8 discordant results), corresponding to making the correct clinical decision less often (97.3\% (vDBS) and 95.3\% (fsDBS)).

\section{Comparison of vDBS and fsDBS}

Five-hundred forty-seven paired vDBS and fsDBS specimens were obtained, including the $149 \mathrm{vDBS} / \mathrm{fsDBS}$ pairs discussed above. Agreement for vDBS and fsDBS, in which both vDBS and fsDBS had concordant detectable or undetectable results, was $86.8 \%$ (Table 2c). $75 \mathrm{vDBS}$ and 78 fsDBS had detectable viremia (>2.74 log copies $/ \mathrm{ml})$. Of specimens with detectable VL, $92.0 \%$ (69) of vDBS and 84.6\% (66) of fsDBS were $>3.0 \log$ copies $/ \mathrm{ml}$. $45.3 \%$ (34) vDBS and $39.7 \%$ (31) fsDBS specimens were $>3.70 \log$ copies $/ \mathrm{ml}$. Median VL was 2.44 [IQR $2.44-2.44$ ] for both vDBS and fsDBS, demonstrating the small subpopulation with detectable VL. Among patients with detectable VL, median VL [IQR] was $3.42[3.17-4.45]$ and 3.29 [3.08 - 4.40], for vDBS and fsDBS, respectively.

There was moderate correlation between vDBS and fsDBS: $\beta=0.88, R^{2} 0.73$, $(p<0.0001)$ (Figure 1c). The mean difference between vDBS and fsDBS was 0.009 log copies/ml [SD: $0.30]$ and was not statistically significant according to the Wilcoxon signed-rank test $(\mathrm{p}=0.267)$ (Figure 2c). 
Among the 547 participants with vDBS and fsDBS specimens, overall clinical concordance at 5000 copies/ml was $98.7 \%$. Overall concordance between vDBS and fsDBS at the 1000 copies/ml threshold was $88.8 \%$.

\section{Discussion}

Despite rapid expansion of access to ART in resource-limited settings, appropriate treatment monitoring strategies remain largely unavailable. The consequence of inadequate monitoring may manifest in accumulation of drug resistance mutations, compromising the effectiveness of future ART options. Plasma-based virologic monitoring is prohibitively complex and expensive for routine use in resource-limited settings. DBS have been suggested as an alternative given the relative simplicity of card preparation and the lack of cold-chain requirements. However, the use of DBS for VL monitoring as collected and prepared in uncontrolled, real-world field conditions is still in its infancy. Furthermore, most previous investigations have relied on venipuncture for DBS card preparation, a potential barrier for implementation in more remote, understaffed clinics. In the present study, we found that DBS obtained from fingerstick prepared by non-laboratory personnel in an uncontrolled setting compared well to both venous DBS as well as plasma for identifying virologic failure of ART patients.

Using the threshold of virologic failure as $>5,000$ copies $/ \mathrm{ml}$, we observed excellent clinical concordance between DBS specimens and plasma: $98.7 \%$ of vDBS and $98.0 \%$ of fsDBS yielded identical clinical decisions as plasma. Very little data exist on the reliability on DBS from fingerstick ${ }^{29}$ : our evaluation of fsDBS confirmed acceptable correlation when compared to plasma. Comparing fsDBS to vDBS, we observed $88.8 \%$ and $98.7 \%$ overall agreement at 1000 and 5000 copies/ml thresholds, respectively.

We observed $100 \%$ sensitivity for both vDBS and fsDBS, compared to plasma, at failure thresholds of 1,000 copies $/ \mathrm{ml}$ and 5,000 copies/ml. Specificity improved at the higher threshold for both specimens. This suggests DBS is highly feasible under current WHO guidelines. However, at the lower threshold, DBS had lower positive predictive values thereby potentially allowing premature switching to $2^{\text {nd }}$ line therapy. Given the higher cost of $2^{\text {nd }}$ line therapy and general absence of $3^{\text {rd }}$ line options, premature switching may have long-term health consequences for the individual and cost considerations for the overall program. Similar studies using the NucliSENS platform have observed lower sensitivity but perfect specificity, a difference that may be due to NucliSENS only detecting HIV RNA while Abbott will detect plasma RNA as well as intracellular HIV RNA and proviral DNA $^{29}$.

Importantly, less than 7\% of all patients tested had viral loads $>5,000$ copies $/ \mathrm{ml}$ and over $85 \%$ of patients were below the limit of detection. This failure prevalence is similar to that observed elsewhere in Malawi-based studies ${ }^{29}$. Only $5 \%$ of plasma samples were between 40 and 550 copies $/ \mathrm{ml}$, suggesting that the higher limit of detection for DBS compared to plasma may not be of great clinical significance. 
DBS VL estimations were universally higher than plasma. This is likely a reflection of proviral DNA and cellular RNA contributions in DBS specimens that artificially raise the VL values. Some have suggested use of DNase pre-step or DNase-containing filter paper to select for $\mathrm{RNA}^{37,47}$. We observed good clinical concordance at 5000 copies/ml failure threshold without using DNase treatment, but additional pre-treatment steps to extract only RNA may be necessary at lower failure thresholds. Abbott's software-imbedded conversion factor accounts for the amount of blood per spot (based on absorbency of DBS cards), the number of spots, the volume of spot elution buffer used per spot, the volume of spot eluate that is processed, and hematocrit (L. Phillips, personal communication, May 3, 2014). Inaccuracies in this experimentally-determined conversion factor, or substantially different hematocrit between our population and the population used to determine this factor, could contribute to systematically higher DBS VL compared to plasma. However, other contributing elements to this conversion factor were consistent with protocol-defined volumes, including the volume of blood per spot given our use of capillary tubes in both vDBS and fsDBS preparations. The WHO guidelines recognize that DBS-based failure should use a higher threshold than that driven by plasma VL. ${ }^{46}$ Larger studies may be necessary to determine if an alternative platform-specific adjustment factor would improve correlation of plasma and DBS VL.

We observed excellent quality of DBS specimens; fewer than 5 cards were rejected due to poor quality. All cards were rejected within the first two-weeks of study initiation and were generally due to blood spots failing to fill the entire outlined circle on the DBS card. This success may be partially attributed to the two-day study training and refresher trainings conducted at sites, during which staff were instructed on proper lancet, capillary, and DBS card preparation technique. The specimen quality may also be related to our reliance on HSAs and HIV Testing Counselors. Although these personnel are considered in the lower cadres in terms of skilled providers within the Malawi healthcare system, they have extensive experience with fingerstick specimen collection for both point-of-care HIV antibody tests as well as early infant diagnosis.

Our study was one of the first to evaluate feasibility and accuracy of fingerstick DBS for VL monitoring under real-world ART clinic conditions. We used eligibility criteria mirroring Malawi Ministry of Health standards. A limitation of this operational-style research was that our population was comprised of many patients with undetectable viremia, meaning a smaller sample for regression and correlation analyses. Nonetheless, our findings reaffirm the feasibility for fingerstick DBS in terms of agreement with plasma, and demonstrate implementation potential in remote settings.

The use of fingerstick for DBS VL monitoring is a tantalizing opportunity, permitting taskshifting to lower-level providers. Fingerstick sampling may be essential for implementation of DBS in overburdened ART clinics in resource-limited settings. Our findings are promising for the expansion of fingerstick DBS for routine VL monitoring.

\section{Acknowledgments}

We would like to thank the numerous ART patients and providers who made this research possible. In addition, we would like to thank A. Soko, M. Mkandawire, C. McLendon, and E. Stein. 
Funding: This study was funded by Centers for Disease Control and Prevention Public Health Evaluation grant MW.10.1433 and the UNC Center for AIDS Research (P30 AI50410). SER is funded by F30 MH098731-01, T32 GM008719, and R01 AI083059.

Competing interests: SAF has served as an advisory board member for Roche Diagnostics and Hologic Gen-Probe.

Ethical approval: This research was approved by the National Health Sciences Research Committee of Malawi, and the Biomedical Institutional Review Board at University of North Carolina, Chapel Hill. All authors contributed to the development, writing, and/or editing of this manuscript.

\section{References}

1. WHO. HIV/AIDS. WHO; Geneva: 2013.

2. Bertagnolio S, Parkin NT, Jordan M, Brooks J, Garcia-Lerma JG. Dried blood spots for HIV-1 drug resistance and viral load testing: A review of current knowledge and WHO efforts for global HIV drug resistance surveillance. AIDS Rev. 2010; 12:195-208. [PubMed: 21179184]

3. Roberts T, Bygrave H, Fajardo E, Ford N. Challenges and opportunities for the implementation of virological testing in resource-limited settings. J Int AIDS Soc. 2012; 15:17324. [PubMed: 23078767]

4. Calmy A, Ford N, Hirschel B, Reynolds SJ, Lynen L, Goemaere E, et al. HIV viral load monitoring in resource-limited regions: optional or necessary? Clin Infect Dis. 2007; 44:128-34. [PubMed: 17143828]

5. Chaiwarith R, Wachirakaphan C, Kotarathititum W, Praparatanaphan J, Sirisanthana T, Supparatpinyo K. Sensitivity and specificity of using CD4+ measurement and clinical evaluation to determine antiretroviral treatment failure in Thailand. Int J Infect Dis. 2007; 11:413-6. [PubMed: 17331776]

6. Keiser O, Tweya H, Boulle A, Braitstein P, Schecter M, Brinkhof MW, et al. Switching to secondline antiretroviral therapy in resource-limited settings: comparison of programmes with and without viral load monitoring. AIDS. 2009; 23:1867-74. [PubMed: 19531928]

7. Lynen L, Van Griensven J, Elliott J. Monitoring for treatment failure in patients on first-line antiretroviral treatment in resource-constrained settings. Curr Opin HIV AIDS. 2010; 5:1-5. [PubMed: 20046141]

8. Mee P, Fielding KL, Charalambous S, Churchyard GJ, Grant AD. Evaluation of the WHO criteria for antiretroviral treatment failure among adults in South Africa. AIDS (London, England). 2008; 22:1971-77.

9. Meya D, Spacek LA, Tibenderana H, John L, Namugga I, Magero S, et al. Development and evaluation of a clinical algorithm to monitor patients on antiretrovirals in resource-limited settings using adherence, clinical and CD4 cell count criteria. J Int AIDS Soc. 2009; 12:3. [PubMed: 19261189]

10. Palombi L, Marazzi MC, Guidotti G, Germano P, Buonomo E, Scarcella P, et al. Incidence and predictors of death, retention, and switch to second-line regimens in antiretroviral- treated patients in sub-Saharan African Sites with comprehensive monitoring availability. Clin Infect Dis. 2009; 48:115-22. [PubMed: 20380075]

11. Reynolds SJ, Nakigozi G, Newell K, Ndyanabo A, Galiwongo R, Boaz I, et al. Failure of immunologic criteria to appropriately identify antiretroviral treatment failure in Uganda. AIDS (London, England). 2009; 23:697-700.

12. Keiser O, MacPhail P, Boulle A, Wood R, Schechter M, Dabis F, et al. Accuracy of WHO CD4 cell count criteria for virological failure of antiretroviral therapy. Trop Med Int Health. 2009; 14:1220-5. [PubMed: 19624478]

13. Moore DM, Awor A, Downing R, Kaplan J, Montaner JS, Hancock J, et al. CD4+ T-cell count monitoring does not accurately identify HIV-infected adults with virologic failure receiving antiretroviral therapy. J Acquir Immune Defic Syndr. 2008; 49:477-84. [PubMed: 18989232]

14. Meya D, Spacek LA, Tibenderana H, John L, Namugga I, Magero S, et al. Development and evaluation of a clinical algorithm to monitor patients on antiretrovirals in resource-limited settings using adherence, clinical and CD4 cell count criteria. J Int AIDS Soc. 2009; 12:3. [PubMed: 19261189] 
15. Costenaro P, Lundin R, Petrara MR, Penazzato M, Massavon W, Kizito S, et al. Viral load detection using dried blood spots in a cohort of HIV-1-infected children in Uganda: correlations with clinical and immunological criteria for treatment failure. J Clin Microbiol. 2014

16. Bagchi S, Kempf MC, Westfall AO, Maherya A, Willig J, Saag MS. Can routine clinical markers be used longitudinally to monitor antiretroviral therapy success in resource-limited settings? Clin Infect Dis. 2007; 44:135-8. [PubMed: 17143829]

17. Coutinho A, Mermin J, Ekwaru J, Were W, Bunnell R, Kahuaruza F, et al. Utility of Routine Viral Load, CD4 Cell Count, and Clinical Monitoring among HIV-infected Adults in Uganda: A Randomized Trial.

18. Gupta RK, Hill A, Sawyer AW, Cozzi-Lepri A, von Wyl V, Yerly S, et al. Virological monitoring and resistance to first-line highly active antiretroviral therapy in adults infected with HIV-1 treated under WHO guidelines: a systematic review and meta-analysis. Lancet Infect Dis. 2009; 9:409-17. [PubMed: 19555900]

19. Jaffar S, Birungi J, Grosskurth H, Amuron B, Namara G, Nabiryo C, et al. Use of WHO clinical stage for assessing patient eligibility to antiretroviral therapy in a routine health service setting in Jinja, Uganda. AIDS Res Ther. 2008; 5:4. [PubMed: 18307778]

20. Phillips AN, Pillay D, Miners AH, Bennett DE, Gilks CF, Lundgren JD. Outcomes from monitoring of patients on antiretroviral therapy in resource-limited settings with viral load, CD4 cell count, or clinical observation alone: a computer simulation model. Lancet. 2008; 371:144351. [PubMed: 18440426]

21. Schooley RT. Viral load testing in resource-limited settings. Clin Infect Dis. 2007; 44:139-40. [PubMed: 17143830]

22. van Oosterhout JJ, Brown L, Weigel R, Kumwenda JJ, Mzinganjira D, Saukila N, et al. Diagnosis of antiretroviral therapy failure in Malawi: poor performance of clinical and immunological WHO criteria. Tropical medicine \& international health : TM \& IH. 2009; 14:856-61. [PubMed: 19552661]

23. DART. Routine versus clinically driven laboratory monitoring of HIV antiretroviral therapy in Africa (DART): a randomised non-inferiority trial. Lancet. 2010; 375:123-31. [PubMed: 20004464]

24. Badri M, Wood R. Usefulness of total lymphocyte count in monitoring highly active antiretroviral therapy in resource-limited settings. AIDS. 2003; 17:541-5. [PubMed: 12598774]

25. Kantor R, Diero L, Delong A, Kamle L, Muyonga S, Mambo F, et al. Misclassification of first-line antiretroviral treatment failure based on immunological monitoring of HIV infection in resourcelimited settings. Clin Infect Dis. 2009; 49:454-62. [PubMed: 19569972]

26. Jani IV, Peter TF. How point-of-care testing could drive innovation in global health. N Engl J Med. 2013; 368:2319-24. [PubMed: 23758238]

27. Garrido C, Zahonero N, Corral A, Arredondo M, Soriano V, de Mendoza C. Correlation between human immunodeficiency virus type 1 (HIV-1) RNA measurements obtained with dried blood spots and those obtained with plasma by use of Nuclisens EasyQ HIV-1 and Abbott RealTime HIV load tests. J Clin Microbiol. 2009; 47:1031-6. [PubMed: 19193847]

28. Waters L, Kambugu A, Tibenderana H, Meya D, John L, Mandalia S, et al. Evaluation of filter paper transfer of whole-blood and plasma samples for quantifying HIV RNA in subjects on antiretroviral therapy in Uganda. J Acquir Immune Defic Syndr. 2007; 46:590-3. [PubMed: 18193501]

29. Fajardo E, Metcalf CA, Chaillet P, Aleixo L, Pannus P, Panunzi I, et al. Prospective Evaluation of the Diagnostic Accuracy of Dried Blood Spots from Finger-Prick for the Determination of HIV-1 Viral Load with the NucliSENS Easy-Q HIV-1 v2.0 in Malawi. J Clin Microbiol. 2014; 52:1343. [PubMed: 24501032]

30. Glencross DK, Coetzee LM, Faal M, Masango M, Stevens WS, Venter WF, et al. Performance evaluation of the Pima point-of-care CD4 analyser using capillary blood sampling in field tests in South Africa. J Int AIDS Soc. 2012; 15:3. [PubMed: 22284546]

31. Diaw PA, Daneau G, Coly AA, Ndiaye BP, Wade D, Camara M, et al. Multisite evaluation of a point-of-care instrument for CD4(+) T-cell enumeration using venous and finger-prick blood: the PIMA CD4. J Acquir Immune Defic Syndr. 2011; 58:e103-11. [PubMed: 21909029] 
32. Arredondo M, Garrido C, Parkin N, Zahonero N, Bertagnolio S, Soriano V, et al. Comparison of HIV-1 RNA measurements obtained by using plasma and dried blood spots in the automated abbott real-time viral load assay. J Clin Microbiol. 2012; 50:569-72. [PubMed: 22170904]

33. Ouma KN, Basavaraju SV, Okonji JA, Williamson J, Thomas TK, Mills LA, et al. Evaluation of quantification of HIV-1 RNA viral load in plasma and dried blood spots by use of the semiautomated Cobas Amplicor assay and the fully automated Cobas Ampliprep/TaqMan assay, version 2.0, in Kisumu, Kenya. J Clin Microbiol. 2013; 51:1208-18. [PubMed: 23390278]

34. Huang S, Erickson B, Mak WB, Salituro J, Abravaya K. A novel RealTime HIV-1 Qualitative assay for the detection of HIV-1 nucleic acids in dried blood spots and plasma. J Virol Methods. 2011; 178:216-24. [PubMed: 21968095]

35. Johannessen A, Garrido C, Zahonero N, Sandvik L, Naman E, Kivuyo SL, et al. Dried blood spots perform well in viral load monitoring of patients who receive antiretroviral treatment in rural Tanzania. Clin Infect Dis. 2009; 49:976-81. [PubMed: 19663598]

36. Johannessen A, Troseid M, Calmy A. Dried blood spots can expand access to virological monitoring of HIV treatment in resource-limited settings. J Antimicrob Chemother. 2009; 64:1126-9. [PubMed: 19776036]

37. Johannessen A. Dried blood spots in HIV monitoring: applications in resource-limited settings. Bioanalysis. 2010; 2:1893-908. [PubMed: 21083497]

38. Mbida AD, Sosso S, Flori P, Saoudin H, Lawrence P, Monny-Lobe M, et al. Measure of viral load by using the Abbott Real-Time HIV-1 assay on dried blood and plasma spot specimens collected in 2 rural dispensaries in Cameroon. J Acquir Immune Defic Syndr. 2009; 52:9-16. [PubMed: 19620878]

39. Vidya M, Saravanan S, Rifkin S, Solomon SS, Waldrop G, Mayer KH, et al. Dried blood spots versus plasma for the quantitation of HIV-1 RNA using a real-Time PCR, m2000rt assay. J Virol Methods. 2012; 181:177-81. [PubMed: 22401801]

40. Neogi U, Gupta S, Rodridges R, Sahoo PN, Rao SD, Rewari BB, et al. Dried blood spot HIV-1 RNA quantification: a useful tool for viral load monitoring among HIV-infected individuals in India. Indian J Med Res. 2012; 136:956-62. [PubMed: 23391790]

41. Rottinghaus EK, Ugbena R, Diallo K, Bassey O, Azeez A, Devos J, et al. Dried blood spot specimens are a suitable alternative sample type for HIV-1 viral load measurement and drug resistance genotyping in patients receiving first-line antiretroviral therapy. Clin Infect Dis. 2012; 54:1187-95. [PubMed: 22412066]

42. Brambilla D, Jennings C, Aldrovandi G, Bremer J, Comeau AM, Cassol SA, et al. Multicenter evaluation of use of dried blood and plasma spot specimens in quantitative assays for human immunodeficiency virus RNA: measurement, precision, and RNA stability. J Clin Microbiol. 2003; 41:1888-93. [PubMed: 12734222]

43. Hamers RL, Smit PW, Stevens W, Schuurman R, Rinke de Wit TF. Dried fluid spots for HIV type-1 viral load and resistance genotyping: a systematic review. Antivir Ther. 2009; 14:619-29. [PubMed: 19704164]

44. Fiscus SA, Brambilla D, Grosso L, Schock J, Cronin M. Quantitation of human immunodeficiency virus type 1 RNA in plasma by using blood dried on filter paper. J Clin Microbiol. 1998; 36:25860. [PubMed: 9431960]

45. Rottinghaus E, Bile E, Modukanele M, Maruping M, Mine M, Nkengasong J, et al. Comparison of Ahlstrom grade 226, Munktell TFN, and Whatman 903 filter papers for dried blood spot specimen collection and subsequent HIV-1 load and drug resistance genotyping analysis. J Clin Microbiol. 2013; 51:55-60. [PubMed: 23077127]

46. WHO. Consolidated guidelines on the use of antiretroviral drugs for treating and preventing HIV infection. WHO; Geneva, Switzerland: 2013.

47. Viljoen J, Gampini S, Danaviah S, Valea D, Pillay S, Kania D, et al. Dried blood spot HIV-1 RNA quantification using open real-time systems in South Africa and Burkina Faso. J Acquir Immune Defic Syndr. 2010; 55:290-8. [PubMed: 20700058] 

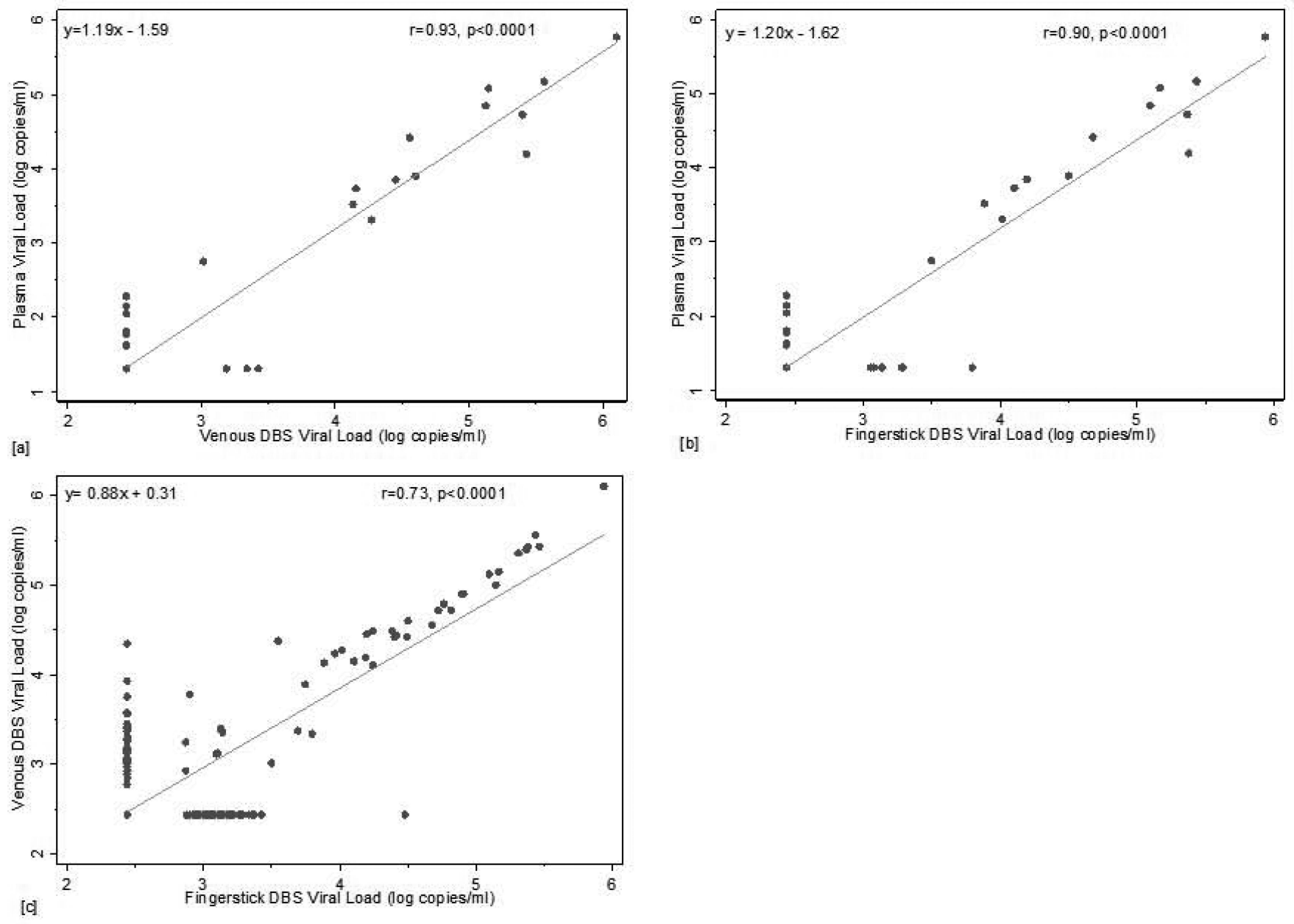

Figure 1.

Linear regression comparing VL values from vDBS (a) and fsDBS (b) to plasma, and fsDBS to $\operatorname{vDBS}(\mathrm{c})$ 

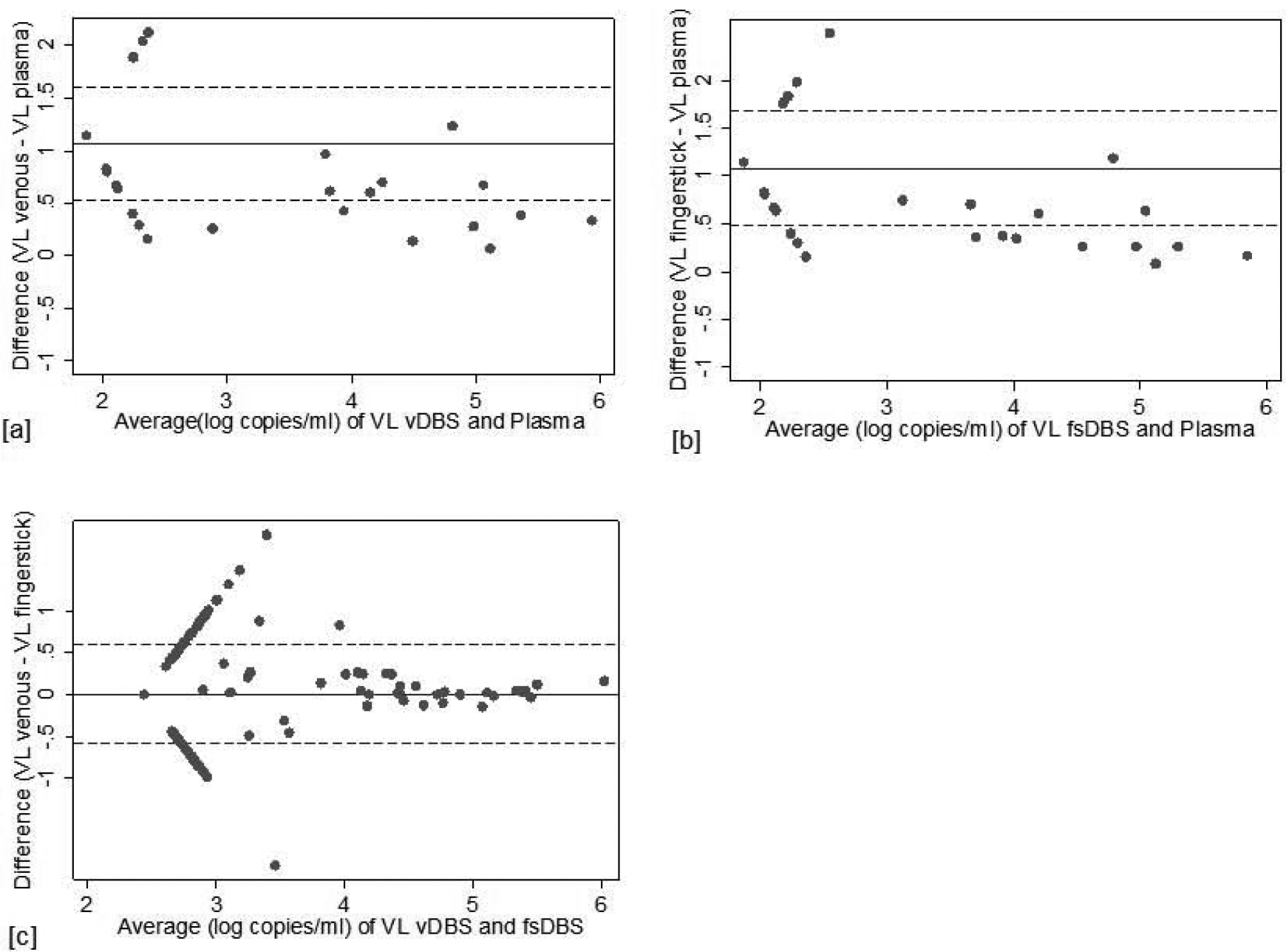

Figure 2.

Bland and Altman plot demonstrating degree of agreement in log copies/ml between vDBS (a), fsDBS (b), and plasma as well as fsDBS and vDBS (c), X-axis plots average VL values for paired specimens. Y-axis represents the difference between the values obtained between the specimen sources evaluated. The solid line represents the mean difference and the dotted lines are the mean $+/-1.96(\mathrm{SD})$. 